\title{
Réflexions sur la pollution cancérigène par les rayonnements ionisants $(*)$
}

\author{
R. LATARJET $(* *)$ \\ (Manuscrit reçu le 23 septembre 1976)
}

\begin{abstract}
RÉSUMÉ
Les effets des faibles doses de rayonnement sur un grand nombre d'individus relèvent du phénomène de la pollution. Ces effets sur l'homme ne sont pas accessibles directement. Il convient donc: $1^{\circ}$ de considérer les études épidémiologiques (l'irradiation cosmique à haute altitude; la radioactivité dans les régions granitiques); $2^{\circ}$ d'extrapoler à partir des résultats des fortes doses; $3^{\circ}$ d'extrapoler à partir des résultats obtenus avec les faibles doses sur les micro-organismes ou les cellules de mammifères in vitro. On dispose également du schéma interpolation, d'ailleurs discuté, d'ABrahamson et al., sur la mutagénèse. Le problème du seuil se situe encore à un niveau théorique, même si la carcinogénèse radio-induite se déroule souvent selon une relation dose-effet avec seuil. Il semble donc utile d'introduire la notion de seuil pratique. De même, c'est sur des considérations génétiques, en particulier, qu'ont été fixées par des organismes internationaux les doses admissibles. Dans le but d'établir des comparaisons quantitatives entre la pollution cancérigène chimique ou induite par les rayonnements ionisants, il serait intéressant d'utiliser la notion de rad-équivalents pour les principaux polluants chimiques mutagènes.
\end{abstract}

\section{ABSTRACT}

The pollution phenomenon groups the effects of small doses of radiation on large populations. These effects on man are not directly accessible. One must: a) consider some epidemiological statistics (cosmic radiation at high altitudes;

(*) Rapport présenté le 20 juin 1975 devant la Commission de l'Énergie du VII ${ }^{e}$ Plan. Les éditions Masson et $\mathrm{C}^{\text {ie }}$ nous ont gracieusement autorisés à reproduire cet article paru dans le Bulletin du Cancer, 1976, 63, $\mathrm{n}^{\circ} 1,1-10$.

(**) Fondation Curie, Institut du Radium, 26, rue d'Ulm, 75231 Paris Cedex 05. 
radioactivity from granitic surroundings); $b$ ) extrapolate from data obtained with high doses; $c$ ) extrapolate from data obtained with low doses on micro-organisms or mammalian cells in vitro. The questionable interpolation scheme of ABRAHAMSON et al. is also available for mutagenicity. The question of a threshold remains theoretical, although radiation-induced carcinogenesis often displays a dose-effect curve with a well marked threshold. A new concept, that of a "practical threshold" is developped, which may be of great usefulness. The main genetic considerations are listed upon which the present international admissible doses are based. Finally, in order to establish quantitative comparisons between chemical and radiation-induced carcinogenic pollution, the concept of "rad equivalents" for the main chemical mutagens is stressed.

Le développement de l'énergie nucléaire de fission et l'inéluctable prolifération des centrales dans le monde au cours des prochaines décennies donnent une dimension nouvelle au problème de la pollution par les substances radioactives et, plus généralement, par les rayonnements ionisants. Parmi les effets redoutés, les cancers humains figurent en bonne place, comme le cas particulier majeur des effets mutagènes s'exerçant au niveau des cellules somatiques. On se trouve ainsi face à une pollution cancérigène bien apte à soulever les plus vives émotions, et à susciter des mouvements générateurs d'une littérature trop souvent dépourvue de l'objectivité nécessaire.

Cette situation m'a inspiré les réflexions de caractère scientifique qui font l'objet de cet article. On remarquera que notre problème s'inscrit dans le cadre plus vaste de la pollution cancérigène et mutagène où la pollution chimique occupe la place la plus importante, et que l'essentiel de ce qui suit s'applique aussi à elle. On ne peut traiter convenablement d'un danger minoritaire en oubliant le danger majoritaire.

On peut dire qu'il y a deux sortes de polluants : les polluants dispensables et les polluants indispensables. Lorsque les premiers soulèvent une difficulté, il suffit de les rejeter. C'est ce qui s'est passé, par exemple, pour le diméthylaminoazobenzène (jaune de beurre) le jour où on s'est aperçu qu'il s'agit d'un puissant cancérigène du foie. Le produit a été interdit dans l'alimentation. Mais on n'agit pas toujours ainsi. J'en veux pour exemple les détergents non biodégradables, qui constituent le principal polluant des eaux fluviales et maritimes côtières, dont on pourrait se passer puisqu'on possède des détergents biodégradables, mais qui subsistent sur le marché par suite de certains avantages économiques qu'ils procurent.

Un polluant peut être considéré comme indispensable dès l'instant que ses avantages l'emportent sur ses inconvénients. Tel est le cas typique des rayonnements ionisants en radiothérapie. On guérit un cancer avec des rayonnements qui peuvent produire des cancers. Mais la probabilité de guérison du cancer en place est en l'occurrence beaucoup plus élevée que celle de la production d'un nouveau cancer. De même, les rayons X dans le radio-diagnostic apportent plus d'avan- 
tages que d'inconvénients. On peut dire qu'un autre polluant indispensable typique est le tabac. puisque la demande l'emporte de beaucoup sur la crainte. Le fumeur continue de fumer bien qu'il sache que sa longévité probable se trouve réduite de 10 ans (ce qui. d'ailleurs. n'est pas mauvais du point de vue démographique).

Le problème devant un polluant indispensable est d'en réduire les inconvénients, c'est-à-dire la nuisance, tout en conservant les avantages du système qui le produit. Le problème se pose alors selon la figure 1 sur laquelle sont portées :

a) la courbe de nuisance en fonction de la dose (dont la forme dépend de l'agent nocif);

b) la courbe donnant le coût de protection pour une réduction de dose donnée.

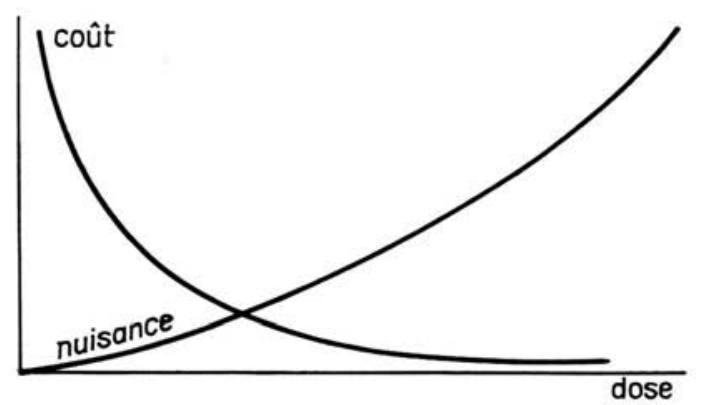

FIG. 1. - Courbe de nuisance et courbe du coût de protection en fonction de la dose, d'après Oftedal [1].

La nuisance augmente évidemment avec la dose. D’autre part, le coût de la protection augmente très vite à mesure que la dose diminue. Il est assez facile de supprimer « le plus gros ». Mais lorsqu'on veut supprimer les dernières traces, cela devient très difficile. Par exemple, les automobiles américaines portent un dispositif réduisant notablement la teneur en hydrocarbures des gaz d'échappement. Ce dispositif ne modifie pas les performances de la voiture et n'en augmente pas sensiblement le prix. Mais si l'on voulait réduire ces carbures par un nouveau facteur 10 , la voiture ne pourrait plus rouler.

Devant ce graphique, l'attitude raisonnable consiste à trouver la meilleure zone de compromis entre :

a) des risques excessifs (qui sont parfois dissimulés ou minimisés);

b) des exigences superflues de protection.

J'ai déjà cité les détergents non biodégradables comme exemple de polluants dont les risques sont sciemment minimisés, et qui sont actuellement utilisés à des doses excessives. 
Inversement, il n'est pas réaliste de réclamer systématiquement des protections supplémentaires. Cette attitude, qui prend parfois l'allure d'une " démagogie de l'apitoiement ", n'est philosophiquement admissible que si elle s'adresse également à toutes les nuisances et à tous les risques, ce qui n'est pas le cas.

On doit distinguer entre les effets des fortes doses sur un petit nombre d'individus, c'est-à-dire les accidents, et les effets des faibles doses sur un grand nombre d'individus, c'est-à-dire la pollution (les fortes doses administrées à un grand nombre d'individus sont des cataclysmes).

Les accidents soulèvent des problèmes médicaux et demandent des mesures de protection qui ressortissent à la sécurité des installations. Ils n'entrent donc pas dans ce propos.

La pollution soulève les problèmes difficiles et complexes des effets des faibles doses. A cet égard je dois dissiper certaines illusions sur le principe des méga-expériences à faibles doses sur les mammifères. En effet, lorsqu'on augmente le nombre des animaux en expérience pour augmenter le nombre des cas apparemment significatifs, on augmente l'inhomogénéité des circonstances expérimentales et, par suite, les fluctuations non contrôlées. Supposons une expérience avec une dose si faible qu'on ne peut attendre qu'un résultat positif sur $10^{4}$ animaux. Si l'on veut relever dix résultats positifs, ce qui est un minimum. il faut opérer sur $10^{5}$ animaux. non compris les témoins. Opérer sur un tel nombre de souris, le mammifère le plus favorable. est impossible de façon convenable. Les paramètres varieront : race. nourriture, âge. logement, technicien, etc... Il existe une expérience célèbre effectuée au cours des années 50 à Oak Ridge par $\mathrm{M}$. et $\mathrm{M}^{\mathrm{me}}$ Russel [2] sur la mutagénèse par rayonnements ionisants chez la souris. Cette expérience a porté finalement sur 70000 souris. Les difficultés surmontées ont été telles que cette expérience n'a pas été reprise. et je doute qu'elle le soit jamais.

\section{Il existe deux types possibles de méga-expériences :}

a) soit les études épidémiologiques aboutissant a des statistiques, quand elles sont bien conduites. Ainsi, par exemple, la statistique des cancers parmi les survivants d'Hiroshima et de Nagasaki a porté sur plusieurs centaines de milliers d'individus. Elle a exigé un travail gigantesque pendant plus de 20 ans, fourni par l'Université et par un Institut spécial créé à cet effet. Un autre exemple, ce sont les statistiques d'hémopathies chez les habitants des hauts plateaux Andins, pour la recherche d'une influence éventuelle du rayonnement cosmique trois fois plus intense que dans la plaine;

b) sur des organismes monocellulaires : bactéries, champignons, levures, cellules en culture in vitro. Là, on peut travailler facilement sur $10^{9}$ individus dans des conditions homogènes, en sorte que des effets se produisant avec une fréquence de $10^{-8}$ sont repérables et dénombrables. 
On peut citer également des expériences de dimension intermédiaire qui sont très utiles, portant sur des graines ou sur de petits animaux comme les insectes (mouche Drosophile) qu'on peut avoir en grand nombre et dans des conditions d'homogénéité satisfaisantes.

Quand on en vient aux effets des faibles doses sur les mammifères, sur l'homme en particulier, et c'est ceci qui intéresse finalement le plus, on est amené à extrapoler les courbes dose-effet à partir des fortes doses, ou à interpoler les résultats obtenus sur d'autres organismes plus simples.

Voici un récent exemple d'interpolation portant sur la fréquence des mutations produites par les faibles doses de rayonnements ionisants. ABRAHAmSON et ses collaborateurs [3] ont constaté en 1973 qu'il existe une relation linéaire entre l'efficacité de l'induction des radiomutations, c'est-à-dire le nombre de mutations par locus génétique et par rad, et la quantité d'ADN (acide désoxyribonucléique) contenue dans le génome haploïde, laquelle est connue. La figure 2 montre que, sur cette courbe. l'homme, qu'on a situé selon la quantité d'ADN de son génome. se place entre la souris et l'orge. On peut en déduire, sur l'axe des ordonnées, que la fréquence des mutations chez l'homme est de $3.5 \cdot 10^{-7}$ par locus et par $\operatorname{rad}\left({ }^{1}\right)$.

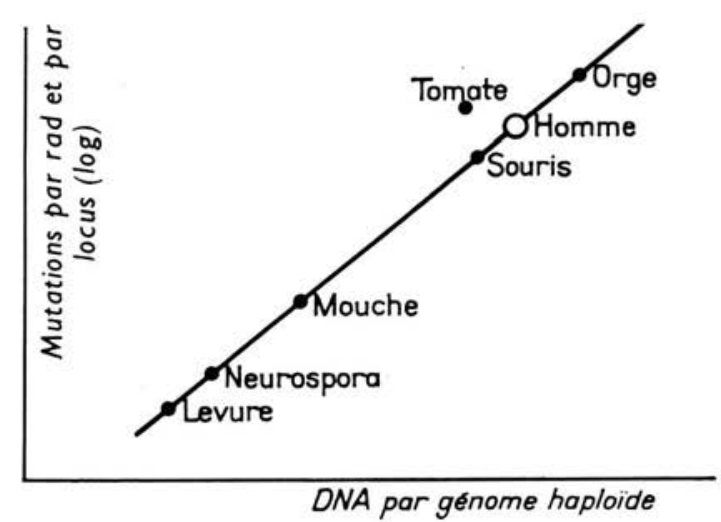

FIG. 2. - Mutagénicité par radiations chez différentes espèces, en fonction de la quantité d'ADN dans le génome haploïde, d'après ABRAHAMSON et al. [3].

(1) La courbe d'ABrahamson est très surprenante. Elle implique soit : a) que le génome entier participe à la mutation d'un locus; soit $b$ ) que lorsqu'on passe d'une espèce à une autre, le nombre des gènes ne change pas, mais leur taille individuelle varie comme la quantité totale d'ADN. Ces deux assertions ne peuvent être facilement admises. Dans une récente publication, Schalet et Sankaranaravanan (Mutation Research, 1976, 35, 34) ont sévèrement critiqué le travail d'ABRAHAMSON et al., en sorte que l'extrapolation interspécifique fondée sur ce travail se trouve remise en question. 


\section{III. - NOTION DE SEUIL}

Lorsqu'on fait agir de faibles doses d'un polluant sur un système biologique, on dit que l'effet produit comporte un seuil lorsque la courbe dose-effet ne commence pas à l'origine, c'est-à-dire qu'il existe une dose seuil au-dessous de laquelle l'agent ne produit aucun effet. Inversement, on dit qu'il n'y a pas de seuil lorsqu'une dose, si faible soit-elle, a une probabilité non nulle de produire l'effet. Cette question est importante car elle gouverne, entre autres, les notions de risque et de responsabilité.

On connaît des effets sans seuil produits par les rayonnements. Telles sont les mutations géniques et chromosomiques produites par les rayonnements et par les mutagènes chimiques; telle est également l'induction lysogénique. réaction assez particulière produite chez certaines bactéries, et qui se traduit par l'émergence d'un virus.

Mais pour les effets somatiques chez les organismes supérieurs, la question n'est pas résolue. Comme on a vu précédemment, l'expérience directe est impossible, et c'est particulièrement vrai dans le cas du cancer dont je parlerai plus loin.

C'est pourquoi je crois important d'introduire la notion de «seuil pratique ". Les effets produits par les polluants existent, en général, à l'état normal avec une certaine fréquence naturelle de base. Les polluants n'interviennent que pour élever cette fréquence. Disons qu'il y a une sorte de bruit de fond en l'absence de pollution. Je définis le « seuil pratique " comme la dose de polluant au-dessous de laquelle aucune augmentation significative de cette fréquence n'est manifeste.

Par exemple. les rayonnements ionisants existent à l'état naturel, soit sous forme de rayons cosmiques, soit sous forme de radioactivité du sol. Le rayonnement cosmique triple entre l'altitude 0 (35 à $40 \mathrm{mrad}$ par an) et l'altitude $3000 \mathrm{~m}$ (90 à $130 \mathrm{mrad}$ par an). En France, les doses du rayonnement naturel total reçues par les gonades de l'homme (compte-tenu de la protection par les tissus qui les recouvrent) sont de 45 à 90 mrem par an dans les plaines alluviales et calcaires, et de 180 à 350 mrem par an dans les régions granitiques, soit un rapport de 4 [4]. Si on compare les populations de ces diverses zones et de très nombreuses études statistiques ont été effectuées dans plusieurs pays on ne trouve pas de différence significative de la fréquence des altérations génétiques ou somatiques au sein des populations. Cette zone de doses apparaît donc comme un seuil pratique au-dessous duquel il ne paraît pas nécessaire de descendre (à cet égard certaines décisions prises récemment aux États-Unis me paraissent excessives, et je doute d'ailleurs qu'elles soient respectées à long terme).

On ne doit d'ailleurs pas exclure que les faibles doses naturelles soient dépourvues d'effets bénéfiques. Ces radiations, au sein du seuil pratique, ont baigné le développement des espèces depuis l'origine; elles n'ont cessé de jouer un rôle dans la sélection, et il faut y réfléchir à deux fois avant de ne leur attribuer que des maléfices. 


\section{IV. - CAS PARTICULIER DU CANCER}

L'expérience directe est ici impossible, et l'extrapolation à partir d'autres espèces est très difficile. Un raisonnement fréquent, mais qui me paraît entaché d'une erreur fondamentale a été souvent développé, à savoir: « La transformation d'une cellule normale en cellule cancéreuse est une mutation (par définition). Ce qui est vrai des mutations l'est aussi de la cancérisation. En particulier, puisqu'il n'y a pas de seuil pour les mutations, il ne doit pas y en avoir pour le cancer. Si on prend pour base la fréquence des mutations et qu'on l'extrapole au cancer. on constate que les doses normales seraient déjà responsables d'une fraction non négligeable des cancers dits spontanés, et que toute augmentation du niveau naturel. si faible soit-elle, serait responsable de nouveaux cancers ".

En fait, il y a là confusion entre la transformation cellulaire maligne, qui est bien une mutation, et le développement clônal de cette cellule transformée pour donner une tumeur. La présence d'une cellule transformée n'est pas un cancer, et c'est fort heureux, car nous sommes tous probablement porteurs de très nombreuses cellules cancéreuses, alors qu'un seulement parmi cinq d'entre nous développera un cancer. Le cancer n'apparaîtra que si une cellule transformée trouve les conditions favorables à son développement, et notamment parvient à vaincre certaines réactions antagonistes de l'organisme. C'est à ce niveau que pourraient intervenir des phénomènes entraînant l'existence d'un seuil.

Il existe de très nombreux systèmes expérimentaux où la cancérisation par radiations ou par produits chimiques se déroule comme s'il y avait un seuil, et même un seuil élevé. Par exemple dans la radioleucémogénèse lymphoïde de la souris sur des lignées favorables [5]:

- 4 irradiations de $175 \mathrm{rad}$ à une semaine d'intervalle ont donné 81 p. 100 de leucémies. la plupart thymiques:

- 2 irradiations de 175 rad à une semaine d'intervalle ont donné 6 p. 100 de leucémies. aucune thymique;

- une seule irradiation de 175 rad n'a provoqué aucune leucémie, soit moins de 1 p. 100.

A Hiroshima. les leucémies au sein de la population humaine qui a survécu aux doses maximales ont augmenté de 7 fois, passant de 2.5 à 17.4 pour 100000 en 1954. date du maximum. Cette augmentation est significative, mais elle est relativement faible si on considère qu'il s'agit des doses maximales. L'augmentation serait de 6 fois après la dose de 100 rad et de 20 fois chez les très rares survivants à la dose de $500 \mathrm{rad}[6]$.

De même, on produit des leucémies chez des sujets ayant reçu des irradiations localisées importantes pour des traitements divers (spondylite ankylosante, hypertrophie du thymus). Dans ces cas, on n'a jamais observé de leucémie lorsque la dose était inférieure a $100 \mathrm{rad}$. La même conclusion a été relevée pour les ostéosarcomes induits par le strontium 90. Dans tous les cas, il semble qu'il y ait un seuil élevé au-dessus duquel la fréquence augmente 
beaucoup plus vite que s'il y avait relation de proportionnalité avec la dose. C'est également le résultat qu'on obtient en considérant les cancers de la peau humaine produits par le rayonnement ultraviolet solaire. Mais ceci n'est pas vrai pour tous les cancers. Ainsi, la production des cancers mammaires chez le rat par les rayons X s'approche beaucoup d'une relation linéaire avec la dose.

N'oublions pas dans tout cela que les hommes développent "naturellement» 20 p. 100 de cancers, c'est-à-dire 200.000 dans une population d'un million, et qu'une augmentation théorique de quelques cas par million ne peut être statistiquement vérifiée.

\section{V. - LES DOSES DE RAYONNEMENT ADMISES}

Le développement de l'énergie nucléaire et les horreurs d'Hiroshima et de Nagasaki ont incité, dès la fin de la Seconde Guerre mondiale, les nations productrices à s'entendre sur la pollution par radiations, et à adopter des limites aux doses de rayonnement dégagées soit par les centrales nucléaires, soit par les déchets radioactifs. Une position très sage et très réaliste a été prise qui a consisté à prendre des décisions rapides bien qu'imparfaites, et à les réviser par la suite en fonction des progrès acquis dans nos connaissances en radiobiologie. Une commission internationale de l'O.N.U. a été chargée de cette responsabilité. C'est ainsi que depuis 1950 , et à plusieurs reprises, cette commission a établi des normes de sécurité qui ont rendu jusqu'ici les plus grands services.

Ces normes ont été fondées sur de nombreuses considérations dont les premières furent les suivantes :

1) Ainsi que je l’ai déjà dit, il faut considérer d'une part les sur-expositions individuelles qui affectent un faible nombre d'individus, et les pollutions diffuses qui en affectent un grand nombre. Les mesures à prendre sont évidemment bien différentes dans les deux cas.

2) En ce qui concerne les irradiations individuelles, on doit se rappeler que les gamètes humains (ovocytes dans les ovaires de la femme, spermatogonies dans les testicules de l'homme) sont arrêtés au cours de la méiose. Ils intégrent donc ce qu'ils reçoivent jusqu'à leur utilisation, c'est-à-dire une conception éventuelle. Il est donc intéressant de considérer la dose de rayonnement accumulée en 30 ans, durée moyenne de cette période d'intégration.

3) La dose de rayonnement qui double la fréquence des mutations spontanées chez l'homme n'est pas directement mesurable. On peut toutefois l'estimer indirectement (voir $\$ 2$ ). On trouve alors qu'elle est comprise entre 30 et 90 rad, selon l'étalement de la dose, soit en moyenne 60 rad. Ceci représente de 20 à 40 fois l'irradiation naturelle accumulée pendant la période de 30 ans (ce qui prouve que la radioactivité naturelle ne joue qu'un rôle faible dans la production des mutations spontanées).

4) On peut prévoir assez bien ce qui se passerait si l'on administrait la dose de doublement d'environ $60 \mathrm{rad}$. Les mutations géniques augmenteraient 
jusqu'à atteindre une fréquence d'équilibre sensiblement double de la fréquence actuelle. S'il s'agit d'un gène récessif abondant dans la population, cette évolution se heurte à une grande inertie, en sorte que le temps nécessaire pour atteindre ce doublement pourrait demander quelques centaines de générations, c'est-à-dire quelques milliers d'années. Cet aspect du problème est donc dépourvu d'intérêt. Si le gène est rare, l'équilibre de fréquence double sera atteint beaucoup plus rapidement, par exemple en une dizaine de générations, soit 250 ans.

Pour les gènes dominants, le doublement de la dose se manifestera beaucoup plus tôt; le doublement des tares pourra être atteint en moins d'un siècle. Mais il y a lieu de considérer que les tares dominantes sont en général bénignes, et que ce sont les tares récessives qui correspondent aux anomalies graves.

En ce qui concerne les mutations chromosomiques, on ne sait pas grand chose, sinon que les chromosomes humains sont très radiosensibles, que leurs cassures ne sont pas défavorables à celui qui les porte, mais le sont pour sa descendance, par les anomalies de la méiose qu'elles entraînent. Ces mutations chromosomiques sont éliminées assez rapidement, en quelques générations, mais leur existence est semée d'individus gravement anormaux.

5) C'est sur ces considérations en particulier, et sur bien d'autres d'ailleurs, que les doses admissibles de rayonnements ionisants disséminées au sein d'une large population ont été fixées à des valeurs qui doublent à peu près l'irradiation naturelle, c'est-à-dire entre 20 et 40 fois moins que la dose de doublement des mutations. Il ne me paraît pas nécessaire d'aller plus loin. En effet, ces doses imposent à cette population une charge génétique très légère qui sera sans doute impossible à détecter. Elle est en tous cas bien moindre que celle qu'apporte la médecine d'aujourd'hui. Celle-ci, en s'opposant à l'élimination naturelle des individus génétiquement tarés, permet à ceux-ci de se reproduire et d'étendre leurs tares avant de disparaître eux-mêmes. D'autre part. les rayonnements ont pris une avance chronologique considérable sur la pollution chimique en ce qui concerne l'adoption de doses admissibles. et il convient maintenant de savoir où nous en sommes de la pollution chimique mutagène.

\section{VI. - POLLUTION CHIMIQUE MUTAGÈNE ET RAD ÉQUIVALENTS}

Dans le cadre de la production d'énergie. la pollution chimique mutagène est un sous-produit quantitativement plus important que la pollution par les rayonnements. Les combustions dégagent en quantités non négligeables des substances telles que le formaldéhyde et les hydrocarbures polycycliques cancérigènes qui lèsent le matériel génétique des cellules vivantes. Aussi conviendrait-il d’établir des doses admissibles pour ces polluants chimiques, comme on l'a fait pour les rayonnements. Des perspectives viennent de s'ouvrir 
qui vont permettre peut-être de procéder rapidement. et de rattraper les rayonnements en quelques années.

En effet. ces substances agissent au niveau du matériel génétique. et y produisent des altérations très semblables à celles que produisent les rayonnements. Tellement semblables même que les cellules réagissent à ces lésions comme à celles des rayonnements par la mise en action de systèmes de réparation qui sont sans doute les mêmes.

$\mathrm{Si}$, sur un système biologique donné, on applique une quantité donnée d'un polluant chimique de ce type, on peut en dénombrer les conséquences (mort, mutations) et surtout dénombrer les lésions sur le matériel génétique. On peut alors déterminer la dose de rayonnement qui. sur le même système, produirait le même nombre de lésions; c'est-⿳亠丷⿵-dire accrocher à la dose du produit chimique une dose de rayonnement " équivalente ".

$\mathrm{Si}$ aucune mauvaise surprise ne survient dans ces recherches, on pourra dans quelques années connaître les " rad équivalents " pour les principaux polluants chimiques mutagènes et, par ce biais, leur extrapoler les doses admissibles déjà adoptées pour les rayonnements.

Dans ce domaine je conseille de procéder. comme on l'a fait pour les rayonnements, avec réalisme et modestie. c'est-à-dire procéder par étapes, en prenant des décisions que l'on sait imparfaites, et en les améliorant progressivement à mesure que nos connaissances se précisent.

\section{BIBLIOGRAPHIE}

[1] Oftedal P. Medical and industrial exposure in relation to genetic risk. Curr. Top. Rad. Res., 1973, 9, 66-71.

[2] Russell W. L., Russel L. B. Dependence of mutation frequency on radiation dose rate in female mice. Proc. nat. Acad. Sc. (Wash.). 1959, 45, 18-23.

[3] Abrahamson S., Bender M. A., Conger A. D., Wolf S. Uniformity of radiation-induced mutation rates among different species. Nature (Lond.), 1973, 245, 460-461.

[4] Voir notamment les rapports du Comité d'Experts des Radiations à l'O.M.S. n 166,1959 et $\mathrm{n}^{\circ} 248,1962$.

[5] Duplan J. F., Latarjet R. Studies on the mechanism of radiation-induced leukemogenesis in C57BL mice. Cancer Res., 1966, 26, 395-399.

[6] Rossi H. H., Kellerer A. M. The validity of risk estimates of leukemia incidence based on japanese data. Radiat. Res., 1974, 58, 131-140. 